\title{
Relations between hypometabolism in the posterior association neocortex and hippocampal atrophy in Alzheimer's disease: a PET/MRI correlative study
}

\author{
K Meguro, C LeMestric, B Landeau, B Desgranges, F Eustache, J-C Baron
}

\begin{abstract}
Objectives-Hippocampal atrophy and hypometabolism in the posterior association neocortex are two well known features of Alzheimer's disease. A correlation between these two features was reported twice previously, suggesting intriguing relations. This question has been reassessed, this time controlling for severity of dementia as well as assessing each side of the brain separately and using a voxel based image analysis in addition to the previously employed regions of interest (ROIs).
\end{abstract}

Patients and methods-Eleven patients were studied with probable Alzheimer's disease and mild to moderate dementia in whom both volume MRI and PET assessed cerebral glucose consumption (CMRGlc) were available. Hypothesis driven correlations between hippocampal width (an index of atrophy) and CMRGlc were performed for two posterior association regions, the superior temporal and the inferior parietal (angular gyrus) cortices, using ROIs set separately for each hemisphere. To confirm significant correlations from the ROIs approach, if any, and to assess their specificity for the posterior association neocortex, CMRGlc image voxel based analysis of correlations with hippocampal width was then carried out. Results-There was a significant correlation, in the positive-neurobiologically expected-direction, between right hippocampal width and right angular gyrus metabolism ( $p<0.01$, Spearman), which remained significant with Kendall partial correlation controlling for dementia severity (estimated by mini mental state scores). Statistical non-parametric mapping (SnPM) confirmed this correlation $(p<0.025)$, and showed a single additional correlation in the right middle temporal gyrus $(p<0.005)$, which is also part of the posterior association cortex.

Conclusion-The findings with both ROIs and voxel based mapping replicate earlier reports of a relation between hippocampal atrophy and ipsilateral association cortex hypometabolism in Alzheimer's disease, and for the first time document that this relation is both region specific and independent of the dementing process itself. Why the correlation was significant only for the right hemisphere is unclear but may be related to the limited sample. Hippocampal-neocortical disconnection due to early and severe medial temporal lobe pathology may at least partly explain the posterior association cortex hypometabolism found in Alzheimer's disease. (f Neurol Neurosurg Psychiatry 2001;71:315-321)

Keywords: hippocampus, glucose utilisation, PET, Alzheimer's disease

In Alzheimer's disease, two well known findings with structural ${ }^{12}$ and functional ${ }^{34}$ neuroimaging are hippocampal atrophy and glucose hypometabolism in the posterior association cortex, respectively; furthermore, both can be documented even at early stages of the disease. ${ }^{56}$ By contrast with hippocampal atrophy, which clearly results from local neuronal pathology, ${ }^{1}$ the mechanisms underlying glucose hypometabolism in the posterior cortex remain debated. Thus, although neurofibrillary tangles and amyloid plaques do affect the posterior association cortex, ${ }^{78}$ the few studies that have assessed the relations between posterior cortex hypometabolism and local pathology have reported inconsistent and at best weak correlations. ${ }^{9-13}$ This has led to the alternative hypothesis that hypometabolism in the posterior association cortex may at least in part result from disconnection effects due to neuronal lesions in remote but connected areas. ${ }^{411}$ Specifically, as the mesial temporal region exhibits prominent and early neuronal pathology in Alzheimer's disease ${ }^{14} 15$ and has strong reciprocal connections with the association areas, ${ }^{16}$ hippocampal damage would be a plausible source of remote neocortical effects. ${ }^{11}$ This hypothesis has recently received strong supportive evidence from combined lesional and PET studies in both the rat and the baboon. ${ }^{17} 18$

One straightforward way to test this hypothesis is to assess the correlations between the magnitude of posterior cortex hypometabolism and the degree of hippocampal atrophy. In patients with probable Alzheimer's disease, Jobst et $a l^{19}$ reported a significant correlation between side to side asymmetry in hippocampal thickness and corresponding asymmetry scores in posterior cortex hypoperfusion, assessed by CT and SPECT, respectively. Subsequently, Yamaguchi et $a l^{20}$ replicated these findings in a group of 13 patients with Alzheimer's disease using $1.5 \mathrm{~T}$ T1 weighted 
MRI to assess hippocampal thickness and PET derived brain glucose consumption (CMRGlc) to assess synaptic function. However, neither of these two studies controlled for the potentially confounding effect of overall disease severity (according to which two causally unrelated phenomena caused by a single disease process may be coincidentally correlated). In addition, Jobst et $a l^{19}$ used only a visual (subjective) assessment of their SPECT data, whereas Yamaguchi et $a l^{20}$ did use regions of interest (ROIs) but without assessing each side of the brain separately, an important consideration in relation to the hippocampus-neocortex connection hypothesis. Furthermore, in neither study were the functional brain images evaluated in a systematic and objective way as afforded by voxel based analysis. ${ }^{21} 22$

The aim of the present study was therefore to test the replicability of the findings of Jobst et $a l^{19}$ and Yamaguchi et $a l^{20}$ by means of measurements of CMRGlc and hippocampal atrophy, using an approach similar to that employed by these authors but including the following novel features: (1) assessing each hemisphere separately; (2) performing partial correlations to control for the potentially confounding effects of disease severity; and (3) assessing the CMRGlc-hippocampal atrophy relations on a voxel by voxel basis.

\section{Methods}

PATIENTS

Out of the population of 19 patients with probable Alzheimer's disease described by Desgranges et $a l,{ }^{21}$ a three dimensional MRI study allowing reslicing the data for optimal measurement of hippocampal atrophy was available in 11 patients (six men and five women), aged 61-79 years; they were all right handed and had sporadic disease. The diagnosis was based on thorough clinical, neuropsychological, and biological as well as neuroradiological investigations, and all patients fulfilled the NINCDS-Alzheimer's disease RDA criteria for probable Alzheimer's disease. ${ }^{23}$ They all had significant episodic and working memory impairment, and all but one had significant semantic memory impairment (assessed by category fluency). All the patients were studied within 5 years of onset of symptoms and showed mild to moderate dementia, with mini mental state examination $(\mathrm{MMSE})^{24}$ scores ranging from 16 to 23 . In no case did standard brain MRI show evidence of brain infarction or extensive white matter hyperintensities, nor did follow up produce evidence for other degenerative disorders such as frontotemporal dementia or Lewy body disease. The patients did not receive any medication known to affect brain metabolism, and especially acetylcholinesterase blockers were not given before the PET study. No patient had a contraindication to performing an MRI, and all were cooperative for both the PET and the $\mathrm{MR}$ procedures. The imaging procedures were approved by the ethics committee of Caen, and informed consent was received from all the patients and their families.
MRI

The MRI examination was performed using a GE Signa 1.5 T scanner. In addition to standard T2 weighted imaging, a T1 weighted volume was acquired (SPGR with 128 contiguous $1.4 \mathrm{~mm}$ thick coronal cuts).

The SPGR MRI sets were transferred into a dedicated workstation and processed according to customised in house software ${ }^{2526}$ which automatically edits the brain tissue from the surrounding bony and soft tissues, locates the anterior and posterior commissures (AC and $\mathrm{PC}$ ), and places the $\mathrm{x}, \mathrm{y}$, and $\mathrm{z}$ axis of the brain with reference to the AC-PC line. The dedicated three dimensional visualisation software then allowed us to rotate the entire brain in any direction, inspect its surface, and cut it along any axis with the resulting coronal, axial, and sagittal sections appearing in real time on the video monitor. Finally, it was possible to measure any particular distance using a mouse with display of a cursor on the video screen.

To estimate hippocampal atrophy, we used the "minimum thickness" method of Jobst et $a l,{ }^{27}{ }^{28}$ because these authors documented that their measurement was both reproducible and sensitive. ${ }^{2}$ The procedure ran according to these authors as follows: (1) the long axis of the hippocampus was first determined on sagittal planes; (2) the semiaxial plane along this axis was displayed (this plane typically cut through the upper pons); and (3) using the cursor, the thickness of the hippocampus was measured on each side, which represents the "minimum thickness" according to Jobst et al. ${ }^{27}{ }^{28}$ The whole procedure was repeated from the start three times, and the average of the three estimates was calculated for each side. This value is referred to in what follows as "hippocampal width". To account for different brain size among patients, the hippocampal width was divided by the maximal brain width measured in the same coronal plane.

PET

The PET study was carried out according to the standardised procedure used in our laboratory, which has been published in detail elsewhere. $^{2122} 29$ We used a high resolution (intrinsic: $5.5 \times 5.5 \times 9 \mathrm{~mm}, \mathrm{x} \mathrm{y} \mathrm{z}$ ), seven slice tomograph (TTV03, LETI, Grenoble, France). Attenuation correction employed ${ }^{68} \mathrm{Ga}$ transmission scans. The head of the patient was positioned according to Fox's stereotaxic procedure, which uses the glabella $(G)$ and the inion (I) as bony landmarks visible on a lateral $x$ ray film taken while the subject is lying on the PET couch with the head gently secured in a Laitinen frame. The seven PET planes were made parallel to the GI line and lay from -4 to $+68 \mathrm{~mm}$ relative to it (total axial field of view is $81 \mathrm{~mm}$ ); across subjects, the GI line is parallel and lays $21 \mathrm{~mm}$ caudal to the AC-PC line. Plasma curves of the ${ }^{18} \mathrm{~F}$-fluorodeoxyglucose (FDG) were obtained from 24 arterial blood samples collected from the radial artery over 1 hour after the intravenous injection of about 5 $\mathrm{mCi}$ of the radiotracer. The PET images were acquired from 50 to 60 minutes postinjection. Parametric pixel by pixel images of CMRglc 
( $\mathrm{mg} / 100 \mathrm{~g} / \mathrm{min}$ ) were obtained using the autoradiographic method and the FDG rate constants and lumped constant derived from normal subjects. ${ }^{30}$

DATA ANALYSIS

The aim of this study was to look for correlations between the MRI derived measures of hippocampal width and the PET derived measures of CMRGlc.

\section{General strategy}

According to the goals defined in the Introduction, the PET data were analysed in two different ways in successive order. We first applied a hypothesis driven ROI method sampling only the parietotemporal association cortex, the aim of which was to replicate the previously published findings. ${ }^{19} 20$ To objectively confirm the findings from the ROI analysis, if any, and to assess their topographic specificity, a voxel based analysis was then applied. Both analyses were run only on CMRGlc values normalised with reference to the cerebellar vermis (nCMRGlc), a brain region relatively spared in Alzheimer's disease. ${ }^{3}$ This is a classic procedure to reduce the effects of variability in global CMRGlc, ${ }^{21}$ similar to the normalisation of hippocampal width for brain size described above. All the statistical analyses were non-parametric to account for the small sample and to deal with potential non-linearity, non-normal distributions, and outliers.

Region of interest (hypothesis driven) analysis The method for ROI placement has been described in detail by Desgranges et $a .^{21}$ Briefly, the MRI set was first resliced in three dimensions and realigned in the matrix with the PET data with reference to the GI line, using dedicated software. ${ }^{26}$ Then, using the
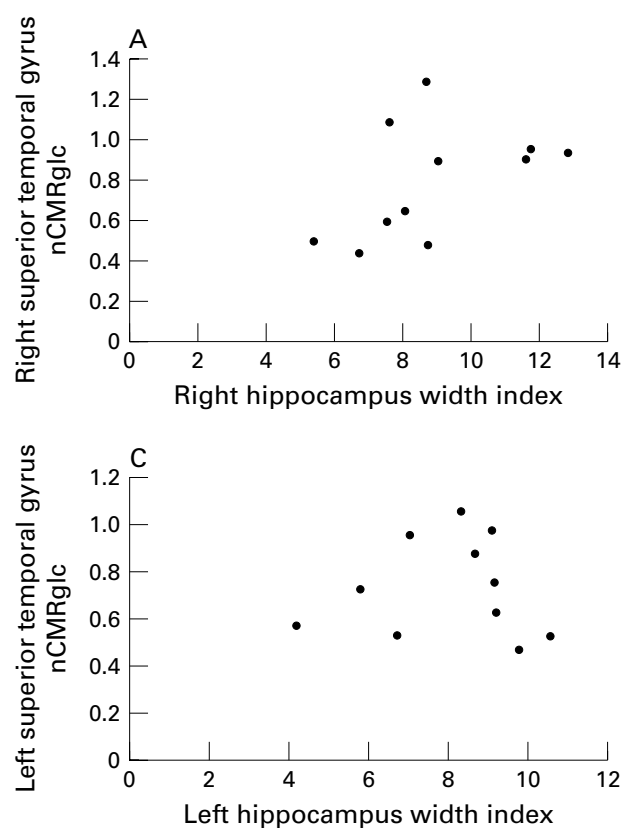

Figure 1 ( $A-D$ ) Plots of the relations between hippocampal width and $n C M R G l c$ for the superior temporal gyrus and the inferior parietal cortex, shown for the right and left hemispheres separately. As assessed by non-parametric Spearman correlation analysis, the positive correlation between right hippocampal width and right inferior parietal cortex $n C M R G l c$ was statistically significant (see table 1 for details).

stereotaxic atlas of Talairach and Tournoux ${ }^{31}$ to identify the anatomical structures, circular (14 $\mathrm{mm}$ diameter) ROIs were first delineated on these coregistered MRI planes and then projected onto corresponding PET planes to obtain CMRGlc values. To test whether hippocampal atrophy was correlated with temporoparietal glucose hypometabolism while keeping to a minimum the number of statistical tests, we constrained the ROI sampling to two areas only on each hemisphere, the superior temporal cortex (Brodmann's area: BA 22/42) with two ROIs, and the angular gyrus (inferior parietal cortex, BA 39) with one ROI. We then obtained the weighted mean CMRGlc across all voxels encompassed by the ROIs for each of these two regions, on each side of the brain. An additional ROI was placed in the cerebellar vermis, to serve as reference for normalisation of the CMRGlc values, as described above.

The relations between hippocampal width and nCMRGlc in these two neocortical regions were analyzed for each side separately, using non-parametric Spearman's correlations. Overall, four correlations only were tested, two for each side. To control for the potentially confounding effect of disease severity, we also performed partial non-parametric correlations (Kendall's $\tau$; running on a SYSTAT software, SYSTAT Inc, Evanstone, IL, USA), using the MMSE score as the index for dementia severity. Statistical significance was set at $p<0.05$ (two tailed).

\section{Statistical mapping}

Apart from the statistical analysis itself, the handling of the CMRGlc images has been described in detail elsewhere. ${ }^{21}$ Briefly, the images were transferred to a SUN workstation and processed with the image analysis software ANALYZE (Biodynamic Research Unit, Mayo
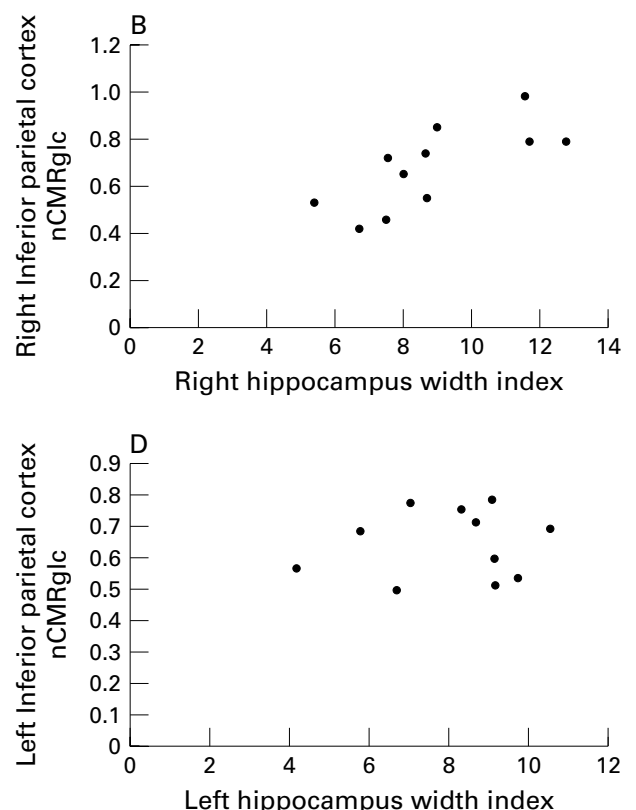
Table 1 Spearman correlations between hippocampal width (HW) and ipsilateral cortical nCMRglc values in 11 patients with probable Alzheimer's disease (see methods for anatomical description of the cortical regions)

\begin{tabular}{lll}
\hline & Right $H W$ & Left $H W$ \\
\hline Superior temporal gyrus & 0.500 & -0.264 \\
Inferior parietal cortex & $0.836^{\star} \dagger$ & -0.018
\end{tabular}

${ }^{\star} \mathrm{p}<0.01$.

$\dagger$ Correlation still significant after covarying the MMS (Kendall's $\tau=0.619, p=0.03$, partial rank order correlation). nCMRGlc $=$ normalised CMRGlc.

Table 2 Positive correlations between right $(R)$

hippocampal width and nCMRglc obtained with SnPM (see methods) in 10 patients with probable Alzheimer's disease. The peaks of significant correlations together with their xyz coordinates in Talairach's space and their $t$ value are given

\begin{tabular}{llllll}
\hline Brain regions & $B A$ & $x$ & $y$ & $z$ & $t$ Value \\
\hline R middle temporal gyrus & 21 & 48 & 2 & 24 & $4.29^{\star \star}$ \\
R angular gyrus & 39 & 36 & 62 & 24 & $3.06^{\star}$
\end{tabular}

${ }^{\star} \mathrm{p}<0.025 ;{ }^{\star \star} \mathrm{p}<0.005$ (two tailed $t$ test).

$\mathrm{BA}=$ Brodmann's area; nCMRglc $=$ normalised CMRglc.

Clinic, Rochester, MI, USA). Manipulations of the image matrix (stereotaxic normalisation) and statistical calculations were carried out with MATLAB (Mathworks, Sherborn, MA, USA). SPM95 (Wellcome Dept of Cognitive Neurology, London, UK) was used for image transformations of this PET data set obtained with the TTV03 PET device. ${ }^{21}$ This entailed masking of the seven PET images by isocontours, interpolation to create a three dimensional space, and spatial transformation to correspond with the 24 axial slices of Talairach and Tournoux. ${ }^{31}$ Because of the limited axial field of view of the PET camera used, the brain could not be studied in its entirety (fig 1). Each voxel obtained from the transformed images represented a parallelepiped with sides of $2 \mathrm{~mm}$ and a thickness of $4 \mathrm{~mm}$. To improve the signal to noise ratio and to limit the effects of interindividual anatomical variations, each image was smoothed using a three dimensional gaussian filter of $16 \mathrm{~mm}$.

As with the ROI analysis, the voxel based statistical analysis was performed with nonparametric statistics. We used the statistical non-parametric mapping software (SnPM, Wellcome Department of Cognitive Neurology, London, UK), ${ }^{32}$ looking for all voxels where nCMRGlc was significantly correlated with hippocampal width. This was done after normalisation of the CMRGlc images by the individual vermis CMRglc. Only positive correlations (in the neurobiologically expected direction) were assessed, using $t>2.752$ $(\mathrm{p}<0.025$, two tailed, uncorrected for multiple tests) as the cut off for statistical significance. To minimise "edge effects" without excluding hypometabolic areas, only those voxels with values greater than $40 \%$ of the whole brain mean were selected for the statistical analysis. ${ }^{21}$

\section{Results}

REGION OF INTEREST ANALYSIS ANALYSIS

As shown in table 1 and illustrated in figure 1, there was a significant positive correlation between right hippocampal width and right inferior parietal nCMRglc $(\mathrm{p}<0.01)$, with a similar but non-significant trend for the superior temporal cortex. The correlations for the left hippocampus were not significant. Partialling out the effect of dementia severity as

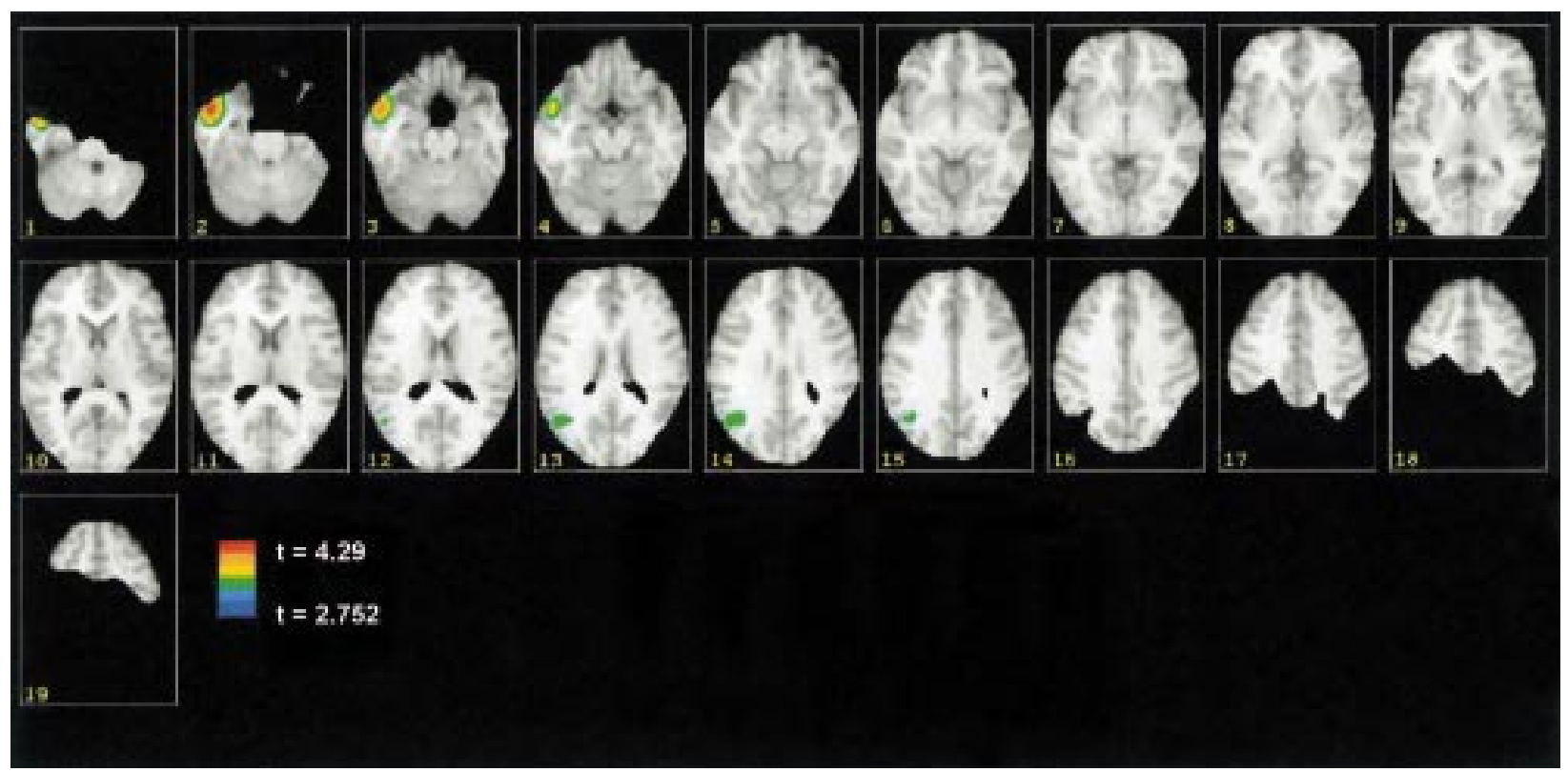

Figure 2 SnPM results of the positive correlations between right hippocampal width and brain glucose consumption ( $n$ CMRGlc, normalised by the cerebellar vermis value). Shown on this figure as coloured areas (projected onto axial T1 weighted MRI cuts from a healthy young subject) are the voxels with significant $(t>2.752,2 p<0.025)$ positive correlation between $n C M R G l c$ and right hippocampal width across 10 patients with probable Alzheimer's disease (see methods for further details). Both the PET data and the standard MRI were spatially transformed with SPM95 to match the 24 planes of the stereotaxic atlas of Talairach and Tournoux. ${ }^{30}$ However, the MRI data set has been cut out to show only the voxels of interest-that is, those where $n C M R G l c$ was $>40 \%$ of the mean value and which were common to all 10 patients, taking into account the limited axial field of view of the PET device used (81 mm). Illustrated here are 19 consecutive axial Talairach planes (every $4 \mathrm{~mm}$ ), from $-28 \mathrm{~mm}$ to $+44 \mathrm{~mm}$ relative to the anterior commissure-posterior commissure plane. The statistic was carried out with SnPM, which calculated non-parametric correlations on a voxel basis. The six level pseudocolour scale ranges from $t=2.752$ to the maximum voxel $t$ value of 4.29. On this display, the right side of the brain is shown on the left side of the images. Two clusters of significant positive correlations between voxel $n C M R G l c$ values and right hippocampal width were found, centred on the right anterior-inferior lateral temporal neocortex (middle temporal gyrus), and the right inferior parietal neocortex (angular gyrus) (see results and table 2 for details). 
reflected by the MMSE scores reduced but did not cancel the significance of the correlation between right hippocampal width and right inferior parietal nCMRGlc (table 1).

VOXEL BASED ANALYSIS

According to our strategy (see methods), SnPM analysis was performed for right hippocampal width only, because only this side showed significant correlations with nCMRGlc data. It was run on 10 patients as the PET data set of one patient was technically inadequate for the SPM spatial transformation. The results are illustrated in figure 2, and the peaks of significant non-parametric positive correlations supplied by this software, together with their Talairach coordinates and their anatomical identification, are listed in table 2 . Significant correlations were found for the ipsilateral middle temporal gyrus and inferior parietal cortex.

\section{Discussion}

SUMMARY OF THE FINDINGS

Our aim in this study was to replicate and expand on previously published findings of a relation between hippocampal atrophy and posterior cortex hypometabolism in Alzheimer's disease. ${ }^{19}{ }^{20}$ Using three dimensional MRI and PET with both the ROI method and voxel based analysis, and considering each side of brain separately, we found a significant and consistent positive correlation between the magnitude of right hippocampal atrophy and the degree of glucose hypometabolism in the ipsilateral temporoparietal association cortex. This correlation did not seem to result simply from a coincidental effect of an underlying disease process as it remained significant even after partialling out for severity of dementia. Furthermore, it was topographically specific for the posterior association neocortex as shown by voxel based analysis of the entire brain parenchyma.

\section{METHODOLOGICAL CONSIDERATIONS}

For the measurement of hippocampal atrophy, several approaches can be used-namely, visual rating, calculation of cross sectional hippocampal area, or actual volumetry. ${ }^{1}$ Although the last is considered the most advanced, it is not exempt from methodological uncertainties. The lack of standard criteria for the boundaries of the hippocampal formation has caused interstudy variability in the reported estimates for hippocampal atrophy in Alzheimer's disease using volumetry. ${ }^{33}{ }^{34}$ Although the minimum hippocampal width measurement method of fobst et $a l^{28}$ may be considered simplistic, it has the advantages of being both straightforward and reproducible, and has been shown to be very sensitive to both normal aging and the Alzheimer's disease process in patients with pathologically proved diagnosis, as well as in the assessment of disease progression in patients with Alzheimer's disease relative to healthy aged controls (see Smith and Jobst ${ }^{2}$ for a review). Our reason for using this method in this work was because we wanted to replicate the earlier findings of Jobst et $a l^{19}$ and
Yamaguchi et al, ${ }^{20}$ who both employed this method.

For PET data analysis, we successively applied two distinct but complementary strategies, according to a well defined plan (see methods). Firstly, so as to limit the number of statistical tests, we employed the classic ROI method to sample a highly limited number of predetermined cortical regions, based on their constant metabolic involvement in Alzheimer's disease and our primary goal of replicating earlier studies. ${ }^{19} 20$ Subsequently, we used a voxel based analysis allowing the assessment of the entire brain in a systematic way, to simultaneously confirm the findings from the ROIs with a more objective method and test their specificity for the posterior cortex. We normalised the CMRGlc values using the cerebellar vermis as reference. We have successfully used this approach in the past to limit the intersubject variance in global metabolism ${ }^{21}$; in addition, normalisation of CMRGlc before the correlation analysis was an appropriate match to hippocampal width, as hippocampal width was also normalised (by brain size; see methods). However, we also reanalysed our data post hoc using absolute CMRGlc values, and found essentially the same results as with nCMRGlc (Spearman correlation coefficients for the superior temporal gyrus and inferior parietal cortex were $0.864, \mathrm{p}<0.001$, and 0.755 , $\mathrm{p}<0.02$, respectively, with right hippocampal width; and -0.109 and 0.082 , not significant, respectively, with left hippocampal width).

Although the sample used was not large, it comprised patients prospectively selected according to strict criteria, in whom it was possible to obtain both FDG-PET and coregistered high quality three dimensional MRI, and whose clinical follow up confirmed the diagnosis of Alzheimer's disease in each case. No other study thus far has reported similarly studied patients. Nevertheless, to account for the risk of non-normal distribution and spurious correlations inherent to limited samples, we elected to use non-parametric statistic in all analyses, whether ROI or voxel based. For the ROI analysis, the significant Spearman correlation obtained for the right angular gyrus was statistically robust $(\mathrm{p}<0.01)$. Furthermore, it was in the neurobiologically expected direction and it replicated earlier findings. These facts altogether argue for the reliability of this finding. For the voxel based analysis, we used SnPM, a method so far little used in clinical research presumably because it is labour intensive and requires intensive computation. In our case, its results were both clear cut and very close to the findings obtained with the ROI analysis, especially the correlation for the right angular gyrus was clearly recovered. Although the ROI method only showed a trend for the superior temporal ROI data only, the SnPM method disclosed a highly significant cluster centred on the right middle temporal gyrus and spreading to the anteriormost part of the superior temporal gyrus (fig 2). Although the region corresponding to this cluster peak was not exactly sampled by our prespecified ROIs, it clearly also belongs to the temporal association 
neocortex, and is usually affected in Alzheimer's disease. ${ }^{35}{ }^{36}$ Finally, the specificity of these correlations for the posterior association cortex was shown by SnPM, which did not show significant peaks elsewhere, even at the lower statistical cut off of $t>2.306, p<0.05$ (data not shown).

For technical reasons, the PET data set of one patient could not be spatially processed with SnPM, thus reducing to 10 cases the sample available for voxelwise correlations. Nevertheless, recalculating the ROI correlations with this reduced sample did not alter the findings (Spearman's $r$ for the right inferior parietal cortex $=0.806, \mathrm{p}<0.01$; Kendall's $\tau=0.662, p<0.02)$.

THE ISSUE OF THE LEFT HEMISPHERE

According to our data, the correlation between hippocampal width and posterior association neocortex nCMRGlc concerned only the right hemisphere. As earlier studies did not analyze the laterality of this relation, ${ }^{19}{ }^{20}$ this surprising finding cannot be compared with previous data. Post hoc SnPM analysis of the relations between left hippocampal width and nCMRGlc did not show any significant cluster of correlation within the left hemisphere, indicating that this negative finding cannot be attributed to data undersampling with the ROI method (data not shown). Although it has been reported that in probable Alzheimer's disease there is a greater metabolic reduction in the left hemisphere, ${ }^{37}$ there was no significant difference in nCMRGlc between the right and left homologous posterior regions in our sample (paired $t$ test; data not shown), indicating essentially symmetric disease. The data plots shown in figure 1, however, suggest that this negative finding may represent a type 2 error due to the limited sample and large intersubject variance. Further studies are necessary to address this intriguing issue.

\section{PATHOPHYSIOLOGICAL INTERPRETATION}

One straightforward hypothesis to explain the correlation between hippocampal atrophy and decreased temporoparietal CMRGlc found in Alzheimer's disease both by us and by previous investigators ${ }^{1920}$ would contend that hippocampal atrophy and decreased temporoparietal CMRGlc are two merely coincidental effects of the pathological process - that is, they are not causally related. To control for this potentially confounding effect, and at variance with these earlier reports, we performed partial correlations using the MMSE score as the index for severity of dementia. Partial correlation is a classic statistical method used to evaluate the influence of a confounder when a correlation is found between two variables. It is particularly useful in clinical research when the underlying disease process may simultaneously induce two distinct clinical features, which in turn may be found significantly intercorrelated without this reflecting a causal, but merely a coincidental, relation. Our finding that the correlation remained statistically significant after partialling out the effect of severity of dementia would therefore argue in favour of the hypothesis that in Alzheimer's disease hippocampal atrophy may contribute to hypometabolism in the posterior association cortex. How could this come about? There are well documented strong reciprocal connections between the hippocampus and the temporoparietal association cortex, ${ }^{16}$ such that neuronal damage in the hippocampus such as occurs in Alzheimer's disease may impair synaptic function in the association neocortex. ${ }^{11}{ }^{12}$ This hypothesis is further supported by recent reports of reductions in neocortical glucose metabolism after lesions of the entorhinal cortex (the obligatory relay between neocortex and hippocampus) in both the baboon ${ }^{17}$ and the rat. ${ }^{18}$ Thus, over and above local Alzheimer's disease pathologywell documented in the posterior association cortex $^{8-15}$-neuronal disconnection from damage to medial temporal lobe structures may therefore partly explain the hypometabolism characteristically found there in Alzheimer's disease, especially in the early stages. Whether hippocampo-neocortical disconnection underlies the cognitive deficits correlated with parietotemporal hypometabolism in Alzheimer's disease ${ }^{2129}$ is another question, but earlier studies suggest that cognitive deficits after subcortical stroke are partly subtended by similarly disconnected networks witnessed by cortical hypometabolism. ${ }^{38}{ }^{39}$ It will be of interest to see if our finding holds in other degenerative dementing processes where hypometabolism ot the posterior association cortex is also reported, such as Parkinson's disease with dementia and dementia with Lewy bodies. $^{4041}$

We thank Dr L Vérard for help with the three dimensional MRI image analysis software, G Perchey for help with the SnPM analysis, Dr MC Petit-Taboué, for help with the PET studies, Dr C Chavoix, for helpful suggestions, and Professor A Dr C Chavoix, for help
Yamadori for his support.

1 De Leon, MJ, Convit A, de Santi S, et al. Structural neuroimaging: early diagnosis and staging of Alzheimer's disease. In: Iqbal K, Winblad B, Nishimura T, et al, eds. York: John

2 Smith Alzheimer's disease, Jobst KA. Use of structural imaging to study the progression of Alzheimer's disease. $\mathrm{Br}$ Med Bull 1996;52:575-86.

3 Foster NL, Chase TN, Fedio P, et al. Alzheimer's disease: focal cortical changes shown by positron emission tomography. Neurology 1983;33:961-5.

4 Friedland RP, Budinger TF, Ganz E, et al. Regional cerebral metabolic alterations in dementia of the Alzheimer's type: positron emission tomography with ${ }^{18} \mathrm{~F}-$ fluorodeoxyglucose. F. Comput Assist Tomog, 1983;7:590-8

Minoshima S, Giordani B, Berent S, et al. Metabolic reducAlzheimer's disease. Ann Neurol 1997;42:85-94

6 Killiany RJ, Gomez-Isla T, Moss M, et al. Use of structural magnetic resonance imaging to predict who will get magnetic resonance imaging to predict who
Alzheimer's disease. Ann Neurol 2000;47:430-9.

7 Haratounian V, Purohit D, Perl DP, et al. Neurofibrillary Haratounian V, Purohit D, Perl DP, et al. Neurofibrillary
tangles in non-demented elderly subjects and mild tangles in non-demented elderly subjects and
Alzheimer's disease. Arch Neurol 1999;56:713-18.

Alzheimer's disease. Arch Neurol 1999;56:713-18.
8 Friedland RP, Brun A, Budinger TF. Pathological and posiFriedland RP, Brun A, Budinger TF. Pathological and posi-
tron emission tomographic correlations in Alzheimer's disease. Lancet 1985;i:228.

9 McGeer PL, Kamo H, Harrop R, et al. Comparison of PET, MRI, and CT with pathology in a proven case of Alzheimer's disease. Neurology 1986;36:1569-74.

10 De Carli C, Atack JR, Ball AMJ, et al. Post-mortem regional neurofibrillary tangle densities but not senile plaque densities are related to regional cerebral metabolic rates for glucose during life in Alzheimer's disease. Neurodegeneration 1992;1:113-21.

11 Mielke R, Schroder R, Fink GR, et al. Regional cerebral glucose metabolism and postmortem pathology in Alzheimer's disease. Acta Neuropathol (Berl) 1996;91:174-9.

12 Mega MS, Chen SS, Thompson PM, et al. Mapping histology to metabolism: coregistration of stained whole-brain ogy to metabolism: coregistration of stained whole-brain
sections to premortem PET in Alzheimer's disease. sections to premortem
NeuroImage $1997 ; 5: 147-53$. 
13 Mega MS, Chu T, Mazziotta JC, et al. Mapping biochemistry to metabolism: FDG-PET and amyloid burden in try to metabolism: FDG-PET and amyloid burd
Alzheimer's disease. Neuroreport 1999;10:2911-17.

14 Braak H, Braak E. Neuropathological stageing of Alzheimer-related changes. Acta Neuropathol (Berl) 1991; 82:239-59.

15 Delacourte A, David JP, Sergeant N, et al. The biochemical pathways of neurofibrillary degeneration in aging and Alzheimer's disease. Neurology 1999;52:1158-65.

16 Lavenex P, Amaral DG. Hippocampal-neocortical interaction: a hierarchy of associativity. Hippocampus 2000 10:420-30

17 Meguro K, Blaizot X, Kondoh Y, et al. Neocortical and hippocampal glucose hypometabolism following neurotoxic lesions of the entorhinal and perirhinal cortices in the nonhuman primate as shown by PET: implications for Alzheimer's disease. Brain 1999;122:1519-31.

18 Hayashi T, Fukuyama H, Katsumi Y, et al. Cerebral glucose metabolism in unilateral entorhinal cortex-lesioned rates: metabolism in unilateral entorhinal cortex-lesioned

19 Jobst KA, Smith AD, Barker CS, et al. Association of atrophy of the medial temporal lobe with reduced blood flow in the posterior parietotemporal cortex in patient with a clinical and pathological diagnosis of Alzheim
disease. 7 Neurol Neurosurg Psychiatry 1992;55:190-4.

20 Yamaguchi S, Meguro K, Itoh M, et al. Decreased cortical glucose metabolism correlates with hippocampal atrophy in Alzheimer's disease as shown by MRI and PET. 7 Neurol Neurosurg Psychiatry 1997;62:596-600.

21 Desgranges B, Baron J-C, de la Sayette V, et al. The neural substrates of memory systems impairment in Alzheimer's disease: a PET study of resting glucose utilization. Brain 1998;121:611-31.

22 Petit-Taboue MC, Landeau B, Desson JF, et al. Effects of healthy aging on the regional cerebral metabolic rate of glucose assessed with statistical parametric mapping. Neuroimage 1998;7:176-84.

23 McKhann G, Drachman D, Folstein M, et al. Clinical diagnosis of Alzheimer's disease: report of the NINCDSADRDA work group under the auspices of department of ADRDA work group under the auspices of department of health and human services task

24 Folstein SE, McHugh PR. Mini-mental state a practical method for grading the cognitive state of patients for the clinician. F Psychiatr Res 1975;12:189-98.

25 Vérard L, Allain P, Travère JM, et al. $3 D$ brain structures extraction using fully automated MRI segmentation. Information processing in medical imaging. Dordrecht: Kluwer Academic Publishers 1995:373-4.

26 Vérard L, Allain P, Travere JM, et al. Fully automatic identification of AC and PC landmarks on brain MRI using scene analysis. IEEE Trans Med Imaging 1997;16:610-16.
27 Jobst KA, Smith AD, Szatmari M, et al. Detection in life of confirmed Alzheimer's disease using a simple measurement of medial temporal lobe atrophy by computed tomography. Lancet 1992;340:1179-83.

28 Jobst KA, Smith AD, Szatmari M, et al. Rapidly progressing atrophy of medial temporal lobe in Alzheimer's disease. Lancet 1994;343:829-30.

29 Penniello M-J, Lambert J, Eustache F, et al. A PET of the functional neuroanatomy of writing impairment in Alzheimer's disease: the role of the left supramarginal and left angular gyri. Brain 1995;118:697-706.

30 Phelps ME, Huang SC, Hoffman EJ, et al. Tomographic measurement of local glucose metabolic rate in humans with (FO-18)2-fluoro-2-deoxy-D-glucose: validation of method. Ann Neurol 1979;6:371-88.

31 Talairach J, Tournoux P. Co-planar stereotaxic atlas of the human brain. 3-Dimensional proportional system: an approach to cerebral imaging. New York: Thieme Medical, 1988.

32 Holmes AP, Bair RC, Watson JDG, et al. Non-parametric analysis of statistic images from functional mapping experiments. F Cereb Blood Flow Metab 1996;16:7-22.

33 Hasboun D, Chantome M, Zouaoui A, et al. MR determination of hippocampal volume: comparison of three methods. AfNR Am ₹ Neuroradiol 1996;17:1091-8.

34 Pruessner JC, Li LM, Serles W, et al. Volumetry of hippocampus and amygdala with high-resolution MRI and threedimentions cies between laboratories. Cerebral Cortex 2000;10:433-42.

35 Kumar A, Schapiro MB, Grady C, et al. High-resolution PET studies in Alzheimer's disease. Neuropsychopharmacology 1991;4:35-46.

36 Jagust WJ, Eberling JL, Richardson BC, et al. The cortical topography of temporal lobe hypometabolism in early Alzheimer's disease. Brain Res 1993;629:189-98.

37 Lowenstein DA, Barker WA, Chang JY, et al. Predominant eft hemisphere metabolic dysfunction in dementia. Arch Neurol 1989;46:146-52.

38 Karbe H, Szelies B, Herholz K, et al. Impairment of language is related to left parieto-temporal glucose metabolism in aphasic stroke patients. F Neurol 1990;237: metabo.

39 Baron JC, Levasseur M, Mazoyer B, et al. Thalamo-cortical diaschisis: PET study in humans. $\mathcal{f}$ Neurol Neurosurg Psychiatry 1999;55:935-42.

40 Van der Borght T, Minoshima S, Giordani B, et al. Cerebral metabolic differences in Parkinson's and Alzheimer's diseases matched for dementia severity. $7 \mathrm{Nucl}$ Med 1997;38:797-802.

41 Ishii K, Imamura $\mathrm{T}$, Sasaki $\mathrm{M}$, et al. Regional cerebral glucose metabolism in dementia with Lewy bodies and Alzheimer's disease. Neurology 1998;51:125-30. 